\title{
FOG-BASED SMART TRANSPORTATION SAFETY IN IOT
}

\author{
Nischitha S, Nethravathi B \\ Department of Information Science and Engineering \\ JSS Academy of Technical Education, Bangalore, Karnataka, India
}

\begin{abstract}
Intelligent/Smart Transportation Security (STS) predicts an expansion in open security through a significant change in perspective for the police power to react from wrongdoing to a proactive one. Applying great police work in STS is essential for the programmed and right recognizable proof of occasions just in case of security dangers in the objective situations. Distributed computing diminishes the costs and high asset utilization of STS frameworks prepared to do great police work, at the cost of presenting extra dormancy through remote brought together frameworks. In this record, the Fog Framework for Intelligent Public Safety in the Transport Environment (FISVER) applies mist processing in a decent video reconnaissance-based STS to apply wrongdoing assurance proficiently. Also, the subtleties of the mishaps and stopping are talked about. Through Fog FISVER, vehicle and haze frameworks bolster self-governing and time wrongdoing identification out in the open transport administrations. A case of a FISVER research center haze test seat was made and broad appraisals were performed on an undeniable test seat. The outcomes show that mist FISVER offers remarkable framework execution and gadget endurance conduct in ordinary STS use cases.
\end{abstract}

Keywords - Smart video surveillance, smart transportation safety, fog computing, ubiquitous computing, Internet of Things (IoT).

\section{INTRODUCTION}

Preventing the death and serious injury from the road accidents is becoming a progressively necessary goal for governments around the world. Rapid advances in data and the communication technology push peoples to move around the urban areas, and as a result, cities that have become overcrowded. The general assembly of the international organization, on 18 April 2018, declared that "the deaths and accidents caused by road accidents continued to represent a serious public health and development problem with the general social and economic adoption of a project. for a resolution entitled as "Improving the international road safety". The supporting documents recommend that Member States that "address road safety globally" implement a discreet road safety management system amongst the alternative initiatives. The availability of the timely assistance for accident victims could be a key request to help minimize the impact of transport accidents. In the Islamic Republic of Pakistan, for example, fifteen teenagers die on average each day due to such accidents. Recent information published by the Statistical Office of the Islamic Republic of Pakistan shows that out of nine, 582 accidents in the year (2016-2017), 4,036 (or fifty-five of the total) resulted in death. Due to the migration of a large population to the cities, the number of vehicles used to travel every day has doubled enormously. Therefore, parking areas in huge cities have been reduced due to the growing range of vehicles. During this state of affairs, it becomes difficult for drivers to look for parking during rush hour in populated cities. many vehicles following identical parking lots create traffic jams. As a result, people pay a lot of their time to find a place to park, which ends in a waste of time, acquisitions of fuel consumption and environmental pollution. Wireless networks, mobile computing, continuous communications, cloud computing, omnipresent / omnipresent processing, etc., Key unit technology activators for innovative services and applications that help ancient cities become smart. Smart Transportation Safety (STS) in the Smart Cities is an attracting specific attention from every analytical and industrial community thanks to the sharp increase in accidents related to vehicle theft, in addition to violent crime on city buses [1]. Authorities often respond to such incidents inefficiently, especially in the developing countries. Historically, police officers respond to the crimes reactively (after the fact) and want population group to be reported in criminal incidents.

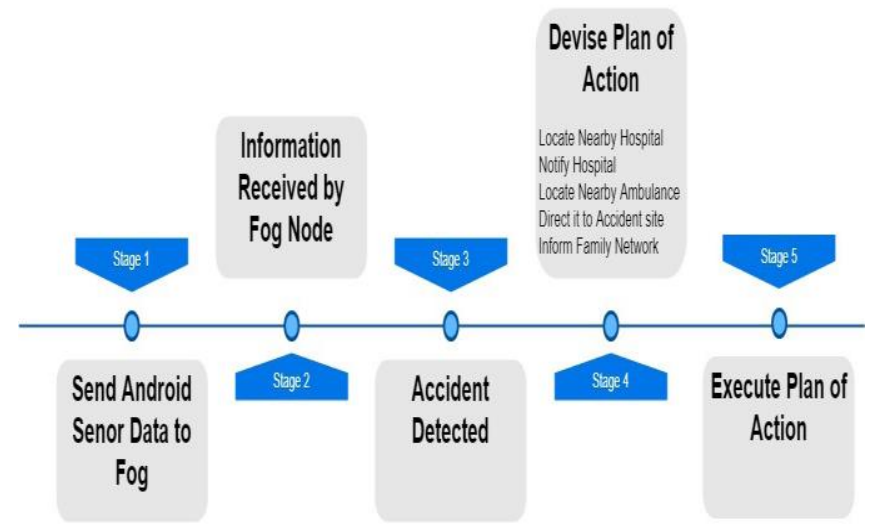

Fig. 1. ERDMS system flow. 


\section{International Journal of Engineering Applied Sciences and Technology, 2020 \\ Vol. 5, Issue 2, ISSN No. 2455-2143, Pages 168-175 \\ Published Online June 2020 in IJEAST (http://www.ijeast.com)}

The police incident response vehicle represents the car used by police officers to refer to a reported crime. Furthermore, the area of the details of the crime unit is sometimes obtained only when the accident occurs, through offline analysis on the video transmissions compiled by the cameras on the bus [2]. Therefore, such a reactive scheme is a obviously not effective in the accident prevention. In addition, there is a high risk of human failure in nervous conditions [3]. STS systems change a paradigm change adopted by the today's police authority to be responded to report crimes, from a reactive model to a prophetic one [4]. The goals of the prophetic model are to anticipate the procedures are taken before the actual event in the plan to prevent criminal accidents on the public buses and reduce deaths. Delaying the emergency reporting of human activities to emergency services will increase the chance of death after a traffic accident. This lawsuit has LEDs for various fashion vehicles that are equipped with integrated emergency response systems to observe accidents and generate acceptable notifications. Such technology has not only become a real possibility worldwide, however, as the Gregorian calendar month 2018, the automated emergency decision system, e-Call, may be legally necessary. reaction to mishaps including more seasoned vehicles and, in those nations, where the establishment of such frameworks isn't required. Moreover, it is additionally amazingly hard to introduce propelled frameworks on more established vehicles. In this unique situation, innovation specifically gave through advances inside the IoT will assume a significant job. Compelling crisis identification and reaction frameworks will assume a key job in decreasing the death toll after an auto collision. Consequently, there is a developing assemblage of examination that proposes answers for the difficulties that emerge in crisis circumstances. A cloud-based occurrence discovery region unit that utilizations cloud assets for preparing, putting away and transmitting information is in the arranging stage. Be that as it may, inactivity is as yet a major issue for huge numbers of the normal arrangements. Analysts have wanted to utilize outer sensors, for example, temperature and stickiness sensors, to identify peculiarities and gather mishap information. While a significant number of the framework territory units are incredibly helpful, the establishment cost on such frameworks can be frightfully high because of the utilization of those costly sensors, making the framework out of reach to low salary clients.

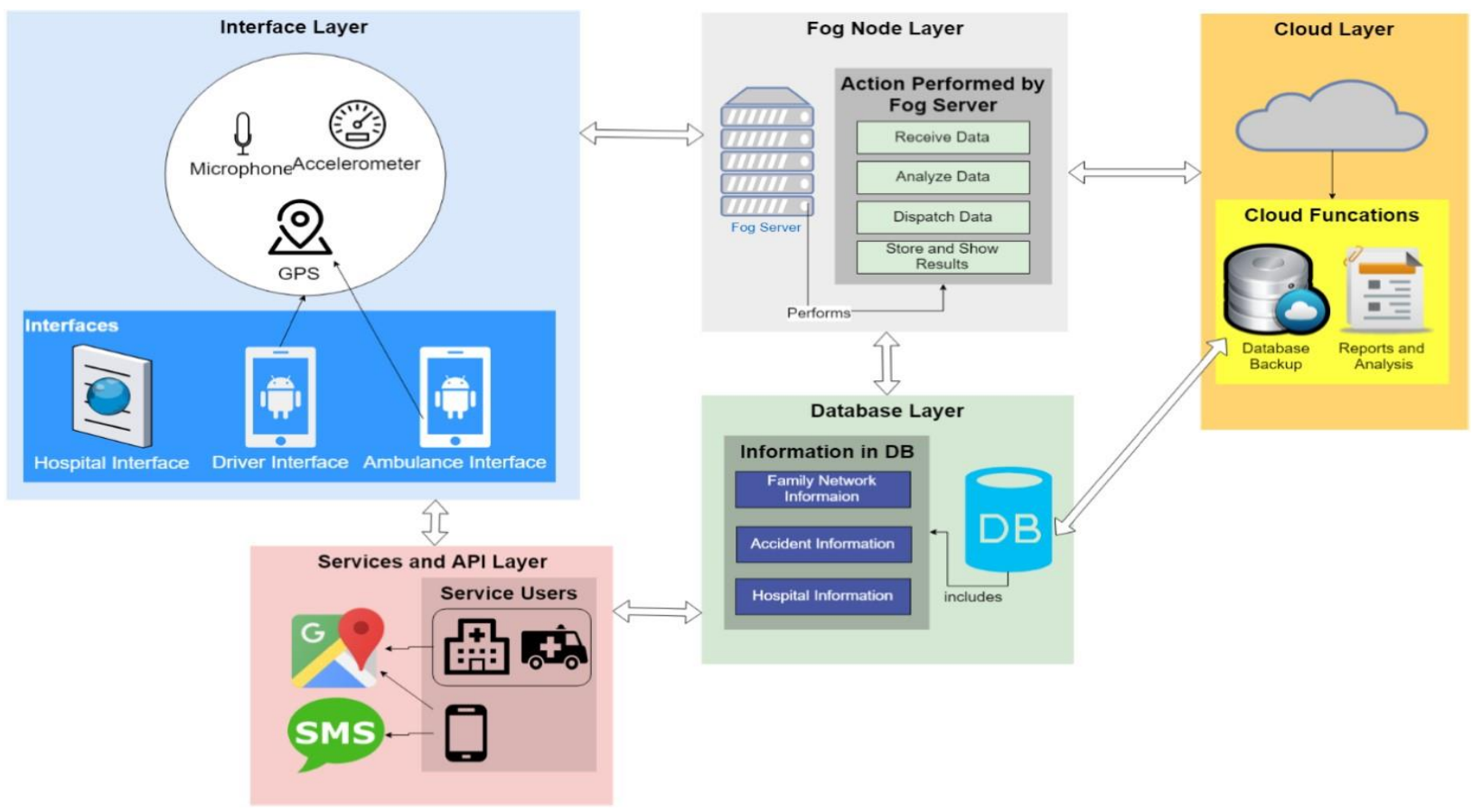

Fig. 2. ERDMS System architecture.

Vehicles that get endorsement inside the universal association (EU) paying little heed to the producer, model or worth class. While these prohibitive advances can encourage the treatment of passing's from mishaps including new vehicles, they can compose a little, low level of vehicles engaged with worldwide mishaps, especially mishaps in creating nations. A test stays an approach to manage the fast
To address the issues referenced above, we will in general propose a haze-based mishap the board framework (we settled on ERDMS). The planned framework intends to decrease reaction and salvage times. The structure of the framework depends on it. The general estimation of the framework is decreased by deceiving the vital sensors of a cell phone to see a partner in a nursing mishap. A cell phone application is built 
up that unites what we need data exploitation of cell phone sensors. Circumstance information is gathered by GPS exploitation. Non-inheritable data is sent to the closest haze hubs for any procedure if essential. In the event that the Nursing mishap partner is known, a close by medical clinic is found and a mishap thought is made to create prompt assistance for mishap casualties. The progression of the ERDMS framework is appeared in Figure 1.

\section{RELATED WORK}

This area audits significant work on STS bespoke assistance applications that exploit touchy police work and investigation strategies. The recommendations profit by cloud-based video police work [8] - [11] which works by steadily transmitting information on police video work cameras to far off cloud foundations for additional examination. Of these occupations, broadband possession takes into account excellent video transmission. Something else, the exactness of identification and acknowledgment exercises running in the cloud can't be ensured. to beat the difficulties presented higher than certain works [12], [13] embrace to lessen the estimation of data because of varieties in video quality, while the framework showed in [14] applies video pressure for transmission. Of these recommendations, the square measure isn't appropriate for use instances of basic dormancy, since the extra idleness brought about by the use of video streamlining innovations (for example pressure, transcoding or resampling occasions) in the responsibility to decrease rates transmission capacity utilization influences video quality. The plausibility of applying digital haze to open police work applications was examined. In these works, haze registering is utilized to process following exercises, speed perception and identification of mishaps that happen in squares and roads [5]. Modern arrangements require cheap cameras that alter groundbreaking picture preparing plans, which trigger moment alarms through email and/or instant messages if a model change is recognized. In spite of the fact that these mechanical arrangements are valuable for watching the fundamental progressing security dangers (for instance, interlopers), it now and then turns into an issue once warnings are sent unremittingly for less imperative explanations behind (model, the location of butterflies or pets), which makes frameworks wasteful.

The accompanying essential standards are significant for arranging the STS arrangement of related sensible and financial police work:

1. Aggieland remedies the recognition and arrangement of criminal occasions by gadgets in the vehicle progressively;

2. Service readiness at mist hubs to guarantee that it is better than supporting basic dormancy applications;
3. Integration with the district's backend frameworks to expand IT at city level; is

4. Crime discovery and anticipating units that have portable preparing for light-footed and customized actuation of occasions.

Disasters are natural or man-made and, in any case, one of the main factors necessary to reduce the loss of human life is time required to respond. There is an over-analysis devoted to solving this problem, given the seriousness of the problem, as well as developments in predictive emergency systems and road safety systems. Recent space analyses include a smartphone-based disaster management system, a cloud-based accident detection and the disaster management system, and also the fog-based disaster management systems.

The cloud-based incident detection and the disaster management system will face the challenges related to latency and information measurement, given that centralized nature of service. A growth thought that can make it easier to deal with these problems is fog computing, which offers these promises of lower latency, quality support, greater resilience and measurability. In addition, using smartphone sensors, emergency detection and management systems are cheaper and easier to implement in historic vehicles. A comparison of the progress of the analysis of smartphone-based incident detection and response systems has been provided, which presents the transitory details of planned solutions, in addition to the limitations of the system. The unit of the document area is sorted by year of publication, in the descending order. Clearly, none of these related works will satisfy the important application needs for following explanations. First of all, the most sensible STS solutions for police work offer archiving and streaming video over time as cloud services, which undoubtedly leads to low lightness rates (due to high latency), survival (consumption energy) and measurability (serious network costs) [6] Furthermore, the transmission network of victimization at the carrier level is incredibly expensive, especially since it is necessary to provide minimum quality guarantees (which is the case to ensure that the 1 video analysis is correct).

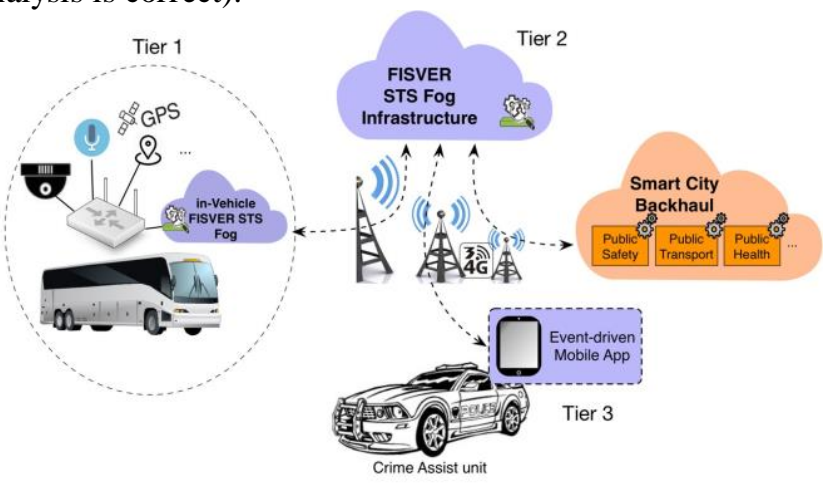

Fig. 3. Illustrating a high-level view of enabled Fog-FISVER ecosystem. 


\section{International Journal of Engineering Applied Sciences and Technology, 2020 \\ Vol. 5, Issue 2, ISSN No. 2455-2143, Pages 168-175 \\ Published Online June 2020 in IJEAST (http://www.ijeast.com)}

We are considering an example of parking to avoid accidents and get good transportation. One of the achievable reasons that will hinder the widespread acceptance of such systems is high latency rate and the network utilization in current cloudbased deployments. For example, a cloud-based technique for good parking is projected in [4]. The authors used sensors to discover parking lots and, the subsequently, location of parking lots has been updated within the cloud. However, vital disadvantages within the methodology is that of promptly changing the position within the cloud in the event that a vehicle enters or exits a specific parking slot following the direct connection of the sensors to the cloud with consequent latency which it is not tolerable for applications and requires a low response time. Therefore, to remedy the shortcomings of the mainly cloud-based strategies in good parking systems, we tend to propose the design of a good parking based mainly on fog. The contributions of this document are triple:

1. The fog-based design for the low-cost parking system was designed for 3-level abuse where the central fog calculation construction is applied. The fog nodes process images of parking lots and then the parking data is displayed on the diode. If a specific car park does not have a free car park, the knowledge of the nearby car parks has been displayed on diode of that parking areas.

2. Projected fog design takes latency and network usage factors into account. Meanwhile, by minimizing latency and network usage, the driving force will select the parking area and park vehicles without gasoline, gasoline and the producing more greenhouse gases. Therefore, it saves the time to identify free parking area and the waiting times for parking following the collaboration of fog nodes that offer knowledge of nearby free parking if the parking is not accessible. very specific parking.

3. The simulations are conducted extensively to evaluate effectiveness and power of projected parking facility enabled for fog. Experimental assessments demonstrate a significant reduction in the latency and the network utilization compared to cloud-based implementations of the good parking systems.

\section{FOG-FISVER FRAMEWORK}

Fog-FISVER aims to produce a fog-compatible replacement thematic field frame (rather than an expensive, high-latency cloud-based approach) for economic support of sensitive video surveillance based primarily on the STS applications. They observe and predict criminal incidents quickly and mechanically, in meantime, keeping a network of coffee in the lead. Within the following subsections, the style details of the square measure of Fog-FISVER design are provided.

\section{Principles of fog-fisver key style -}

Haze FISVER varies altogether from our past work, yet from the related works portrayed in Section II, through the arranging of its structure as indicated by the standards of the haze figuring worldview, with prospect of getting a more prominent effect. in presentation. Regularly, haze count focuses on the edge of the system by displaying every assortment of video information and timespan examination to rapidly meet the low dormancy needs of STS-based video handling through fast access and tangible mindfulness investigation. locally in the mist hub in the vehicle.

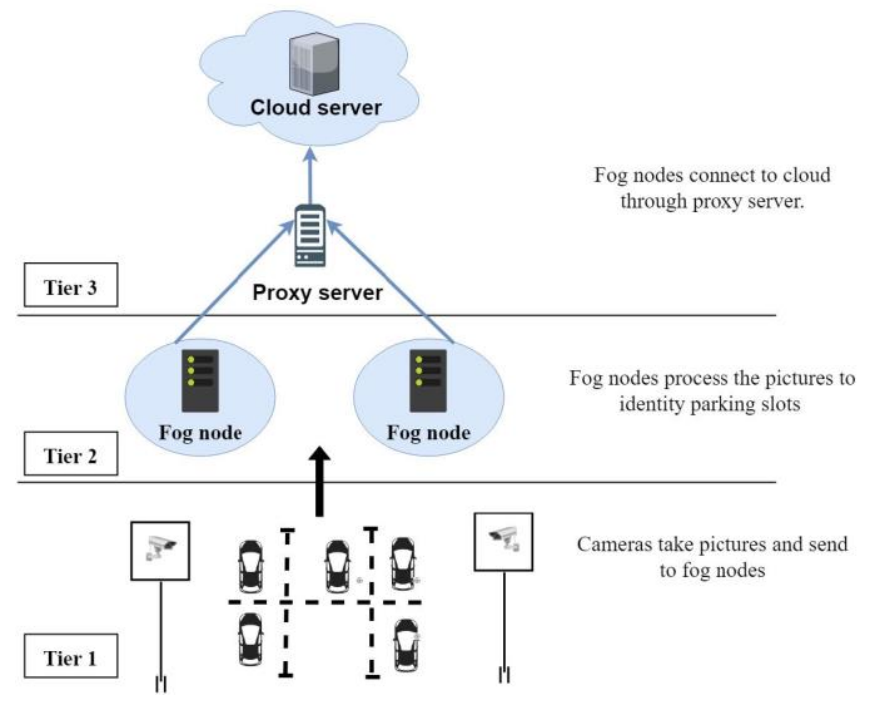

Fig. 4. Fog enabled Smart Parking system's three tier architecture that equipped with cameras and sensors.

Specifically, our past work depends on unified structure in the foundation of information focus to deliver location of criminal dangers as cloud administration, inside which camera in the vehicle should continually transmit information from video to the cloud for the 'investigation. The primary level alludes to the related degree haze hub in the vehicle which incorporates instruments for alluring local tactile mindfulness and the execution of nearby wrongdoing investigation. The subsequent level is acted in the mist processing foundation, examination of superior criminal occasions in the performing expressions for characterization and affirmation of the rate of dangers. The subsequent level additionally looks to discover police episode reaction vehicles that best meet the abilities to rapidly follow a recognized criminal occasion. At last, the third level alludes to portable applications running on cell phones in police occurrence reaction vehicles, which thusly report a wrongdoing to the top client (the cop) at short inactivity interims [7]. 


\section{International Journal of Engineering Applied Sciences and Technology, 2020 \\ Vol. 5, Issue 2, ISSN No. 2455-2143, Pages 168-175 \\ Published Online June 2020 in IJEAST (http://www.ijeast.com)}

\section{Fog part in-vehicle FISVER STS}

Inside the vehicle, a mist hub has the FISVERSTS mist part in the vehicle, which consolidates a gathering of subsystems to screen local wrongdoing dangers over some undefined time frame. Most square highlights measure as follows:

1. Gathering tactile mindfulness inside the transport lodge.

2. Expand the sensorial information on transmission to identify conceivable security dangers.

3. Give criminal data is

4. Actuate the FISVER STS hostile to mist foundation to deliver wrong doing based metadata.

\section{a: Pictures processors}

As a significant part of the structure of the FISVER STS Fog part in the vehicle, the Image Processor module must be arranged as a flexible vision system, conveying the plan to twofold the human vision capacity. Thusly, the style of the Image Processor module should follow electronic picture taking care of advances to convey criminal article disclosure capacities subject to a period of through and through learning [15]. In our exploration community perspective of the FogFISVER structure, the FISVER STS Fog part in the vehicle is completed during a Raspberry Pi propelled PC three B models (1.2 GHz Quad-Core ARMv8 gear, 1 GB of RAM and glint hover card from $64 \mathrm{~GB}$ ) which is outfitted with a first class camcorder [16]. The ability to recognize learning-time objects subject to the significant learning introduced by the image the load up structures is executed in Java (http://java.com) with the help of the incredibly prudent open-source (OpenCV) v3 .1 .0 (http://opencv.org). The limit of the class Stream video, or the library for the most part used for the distinguishing proof and following of articles, is applied to discover the universe of the electronic compass got by picture sensors. We will when all is said in done pressure that this report bases on the FogFISVER framework, along these lines it goes past the purpose of spreading out grow hardware and programming gadgets and approaches, all settled exclusively to allow the creation of research focus models [17]. The structure of the Fog-FISVER sweeping topical camp is expected to coordinate the sensible police work event reliant on STS, with improved system sees similarly as execution [18].

\section{b: Sub part of event dispatcher}

The Event Dispatcher module must be proposed to combine information from each image processor and, thusly, from the units of the vehicle distinguishing part. At the point when an unlawful event is recognized, this information is masterminded into another XML record, which is arranged and sent to the FISVER STS Mist Infrastructure for the resulting level method [21].
The sub-parts of the square of the event dispatcher measure as follows:

1. Event Notifier is charged for amassing data isolated from the enlisted sensor group and sent to the FISVER STS antagonistic to dimness establishment for taking care of. GPS position, vehicle temperature, noxious gases, fires, etc. Square instances of sensors in the vehicle.

2. $\{$ knowledge $\mid$ the information $\mid$ the information $\}$ The widely appealing gatherer exercises between the Event Notifier and the sensors in like manner fill in as applied interfaces to accumulate data from the enlisted sensors.

3. The virtual sensor interface executes a virtual level for data recognizable proof.

\section{FISVER STS fog infrastructure}

The STS Fog Infrastructure fuses Fog-FISVER subsystems that run over the Fog registering substrate.

The administrations and the uses of the FISVER STS Insquare framework are summed up underneath:

1. Models as an assistance, keeping calculations in the vehicle consistently as of not long ago with new meanings of sanctuaries of criminal articles;

2. The order of occasions as insightful PC misuse calculations for the grouping of occasions for a period bolstered the examination of pictures sent by STS haze on the vehicle side (i.e, the occasion dispatcher); e A. J. V. Neto et al.: Criminal help dependent on the haze of touchy IoT traffic.

3. Activating occurrence reaction as administration to discover most appropriate police vehicle and different reports of causal wrongdoings.

\section{a: Coaching of target seeing}

As there is a decent assortment of criminal items, the target objects preparing module permits you to introduce new articles to be perceived. Law requirement specialists (for example a security examiner) will approach just to them, who can embed, refresh or erase meanings of sanctuaries of criminal items. The vast majority of the exercises in the preparation part of the objective articles measure, refresh and remember the meaning of the sanctuary of the criminal items to adjust the cases of STS haze in the vehicle, in which the sub-some portion of the picture processor utilizes them through Start recipe as a parameter to coordinate the criminal article templet [23]. The anticipated methodology makes a record with meanings of templets identified with the wrongdoing object models (for example shading angle, structure design, and so forth.). Templet definitions consolidate a gathering of tests that relate to pictures that 
incorporate criminal items [24]. The PC client can give informational collections identifying with criminal item models and to ceaselessly keep up model definitions to check whether they are perfect with the vehicle's subsystems for the right discovery of criminal mishaps. To do this, the PC client must play out a preparation stage, which can create a related XML record containing meanings of articles illegal. Figure four shows a related case of a templet definition XML document made once as a preparation stage [25]. Endless supply of the technique for characterizing a criminal item definition, the Definitions variants subpart makes a totally new passage for a totally new meaning of a criminal article definition. what's more, stores it in the definition data. Thus, it connects the current model definition with the new article models, another variant recognizable proof code is prepared [26]. The Fog STS in the vehicle conspire inconsistently checks inside the Fog STS FISVER framework whether another form of the sanctuary definitions is advertised.

\section{b: Notification manufacturing plant}

The sub-segment of the warning assembling office is liable for accepting occasion notices sent by all occurrences of the STS mist framework in the vehicle. When a danger occasion has been sent from STS mist subsystems into the vehicle, the occasion arrangement subcomponents approves the got occasion. To guarantee progression of the FISVER STS Fog Subsystems administration, the occasion mediator must work with a procedure line, inside which approaching occasions are gotten and lined for an approval technique that disposes of incorrect occasion messages [27].

\section{c: Mobile applications by event-driven method}

The occasion put together portable applications work with respect to client gadgets (for example Cell phone/tablet in episode reaction police vehicles, outsider security habitats, police authority the executive's places, and so on.) [28] Lightweight methods essentially to get warnings of criminal occurrences and send them beforehand to the cop. To help this, the occasion based versatile application must follow a warning-based way to deal with remain in reserve mode until wrongdoing notices are gotten from the subsystems of the FISVER STS hostile to haze framework et al(2019) [30]. Given the expected benefits of the Fog-FISVER frame elements, a wide variety of applications are often designed to ensure maximum peace of mind. The mobile app was created, designed exclusively to implement the event-based mobile app within the Fog-FISVER laboratory model. A typical NDK state of affairs (https://developer.android.com/ndk) did not often create a native library load for the automata mobile app, created through JNI.

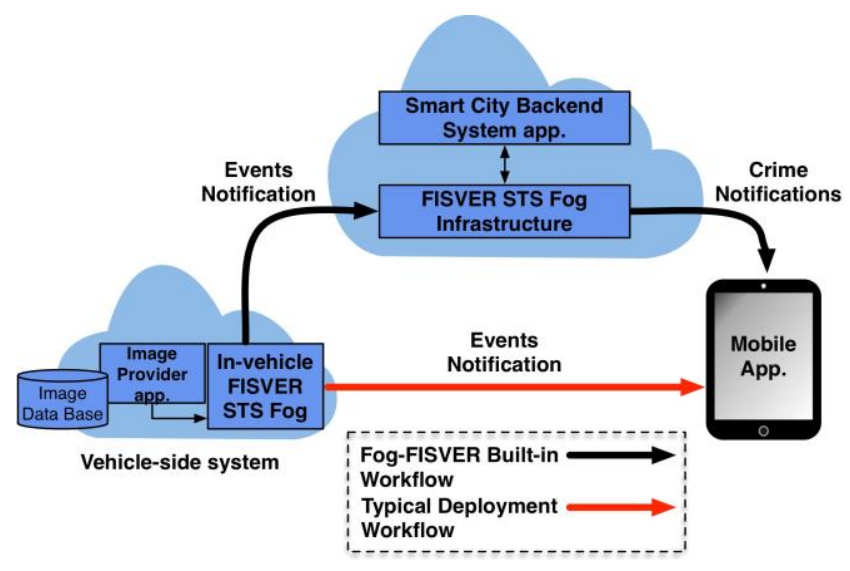

Fig. 5. Fog-FISVER Built-in experiments and Typical Deployment by Testbed configurations used in the evaluations

\section{CONCLUSION}

The principle commitment of this work is that the style, arrangement and investigation of the presentation of an expressive arts structure for the mist colleague to permit the making of good reconnaissance-based STS utilize cases for compelling location wrongdoing in shrewd vehicle frameworks. The framework tends to the STS Fog and STS Fog foundations in the vehicle to improve the exhibition of the STS application [29]. Mist FISVER consolidates a standard component plan that offers extra great strategies available through open web interfaces. The appropriateness and attainability of Fog-FISVER are surveyed by prototyping in the vocal test research facility under the STS use instances of open transport. The aftereffects of the prototyping in the research facility test seat show a critical improvement in equipment execution $(27.76 \%)$, the system $(51.98 \%)$ and vitality sparing force $(62.14 \%)$ contrasted with the conduct of the arrangement of day by day readiness tests [31]. The outcomes uncover that the great use case dependent on STS observation, which fits in with the Fog-FISVER dynamic structure, surpasses all the confinements brought up in Section II, through remarkable framework execution and gadget endurance activities during day by day appropriation. Accordingly, the aftereffect of the readiness of the FogFISVER expressive arts structure in a decent police examination dependent on STS use cases fortifies its fundamental commitment to strategic things by the effect of intensity on the machine, the Value Optimizations networking, framework/gadget endurance and quantifiability.

Future work incorporates learning the effect of abuse utilizing the Fog-FISVER system inside the subsequent deftness between recognizing criminal dangers and versatile application warning. Haze permits a financial and confined information process that can be expressly utilized in time delicate applications. Gives low inactivity edges, geographic 


\section{International Journal of Engineering Applied Sciences and Technology, 2020 \\ Vol. 5, Issue 2, ISSN No. 2455-2143, Pages 168-175 \\ Published Online June 2020 in IJEAST (http://www.ijeast.com)}

appropriation and quality help. Shorter reaction times are viewed as an important solicitation in mishap identification and crisis notice frameworks, as a postponement can prompt death toll. The planned framework conquers the difficulties looked by cloud-based frameworks using distributed computing. The framework created during this work abuses the abilities of cell phones to bring down the value contrasted with comparative OBU-based arrangements.

The framework mutually diminishes the worth and limits by and large inactivity by limiting human mediation through programmed crisis the executives. To exhibit the work, the Associate in Nursing Mechanical Man application was created as a sign of reasoning. The aftereffects of the examination guarantee that mist based ERDMS has lower inactivity, arrange utilization and runtime than cloud-based frameworks.

\section{REFERENCE}

[1] M. A. Hossain (May 2014), "Framework for a cloudbased multimedia surveillance system,' Int. J. Distrib. Sens. Netw., vol. 10, no. 5, (p. 135257).

[2] D.Schonfeld (Sep.2010), "The evolution of signal processing", ,IEEE Signal Process. Mag., vol. 27, no. 5, (pp. 2-6).

[3] Homeland Security News Wire. (Sep. 23, 2011). Study Shows Surveillance Cameras Reduce Crime, in Some Cases.[Online].Available:

http://www.homelandsecuritynewswire.com/study-showssurveillancecameras-reduce-crime-some-cases

[4] G. Karagiannis et al.,(Nov.2011), "Vehicular networking: A survey and tutorial on requirements, architectures, challenges, standards and solutions," IEEE Commun. Surveys Tuts., vol. 13, no. 4,( pp. 584-616).

[5] F. Al-Doghman, Z. Chaczko, A. R. Ajayan, and R. Klempous,(Oct. 2016) "A review on Fog Computing technology," in Proc. IEEE Int. Conf. Syst., Man, Cybern. (SMC), (pp. 1525-1530).

[6] W.ChenandT.Zhang(Mar. 2017), “Fog computing,'’IEEE Internet Comput., vol.21, no. 2, (pp. 4-6) ,VOLUME 6, 2018 11109, A. J. V. Neto et al.: Fog-Based CrimeAssistance in Smart IoT Transportation System

[7] H. Camboim,(2015) "Cloud computing assisted smart surveillance based safe transportation system to improve crime assistance on smart cities,' M.S. thesis, Syst. Comput. Graduate Program, Federal Univ. Rio Grande do Norte, Natal-RN, Brazil.

[8] Y. Wen, X. Yang, and Y. Xu, ( 2010) "Cloud-computingbased framework for multi-camera topology inference in smart city sensing system,', in Proc. ACM Multimedia Workshop Mobile Cloud Media Comput. (MCMC), New York, NY, USA, (pp. 65-70). [Online]. Available: http://doi.acm.org/10.1145/1877953.187797
[9] Y.-T. Cheng, H.-A. Lin, and Y.-J. Yeh, (Sep. 2014) “A flexible architecture of realtime audio transmission to heterogeneous devices for surveillance system,' in Proc. 16th Asia-Pacific Netw. Oper. Manage. Symp. (APNOMS), ( pp. 1-4).

[10] A.K.Paul and J.S.Park,(May 2013), “Multiclass object recognition using smartphone and cloud computing for augmented reality and video surveillance applications,", in Proc. Int. Conf. Inform., Electron. Vis. (ICIEV), (pp. 1-6).

[11] Y. Jian, W. Xin, Z. Xue, and D. Z. You,( May 2015) "Cloud computing and visual attention based object detection for power substation surveillance robots,', in Proc. IEEE 28th Can. Conf. Elect. Comput. Eng. (CCECE), ( pp. 337-342).

[12] D.Rodriguez-Silva,L.Adkinson-Orellana,and F. Gonz'lezCastano, I. Armino-Franco, and D. Gonz'lez-Martinez, (Jun. 2012), "Video surveillance based on cloud storage," in Proc.IEEE $5^{\text {th }}$ Int.Conf.Cloud Comput.(CLOUD),(pp. 991-992).

[13] X. Chen, J.-B. Xu, and W.-Q. Guo,(Jul. 2013) "The research about video surveillance platform based on cloud computing,', in Proc. Int. Conf. Mach. Learn. (ICMLC), vol. 2,(pp. 979-983).

[14] F. Zhu, H. Qiu, and Z. Y. Song,(2013), "Rhizome: A middle-ware for cloud vision computing framework," in Proc. IET Int. Conf. Smart Sustain. City (ICSSC), Shanghai, China, ( pp. 193-197, doi: 10.1049/cp.2013.2023).

[15] N. Chen, Y. Chen, Y. You, H. Ling, P. Liang, and R. Zimmermann, (Apr. 2016), "Dynamic urban surveillance video stream processing using fog computing," in Proc. IEEE 2nd Int. Conf. Multimedia Big Data (BigMM), ( pp. 105-112).

[16] N. Chen, Y. Chen, X. Ye, H. Ling, S. Song, and C. T. Huang,(2017), "Smart city surveillance in fog computing," in Advances in Mobile Cloud Computing and Big Data, vol. 22, C. Mavromoustakis, G. Mastorakis, andC.Dobre,Eds.Cham,Switzerland:Springer,( pp.203226).[Online].Available: http://dx.doi.org/10.1007/978-3-319-45145-99

[17] V. Lanaria. Netatmo Presence Smart Surveillance Camera Can Tell if it's an Intruder or Your Cat Lurking Outside Your Home. Accessed: Jan. 8, 2016. [Online]. Available: http://www.techtimes.com/articles/122385/20160108/neta tmo-presence-smart-surveillance-cameracan-tell-if-it-san-intruder-or-your-cat-lurking-outside-your-home.htm

[18] Skywatch Inc. (2015). Announces Smart Video Surveillance Cloud Service at Cloud Expo. Accessed: Jun.15,2015.[Online].Available: http://www.marketwired.com/press-release/skywatch-incannouncessmart-video-surveillance-cloud-service-atcloud-expo-2015-2029404.htm 
[19] BrickCom IVA Technology. Accessed: Dec. 12, 2016. [Online].

Available:http://www.brickcom.com/products/DetailView .php?series=Other

Applications\&modelname=IVA\#product-features

[20] Smartvue. Accessed: Dec. 12, 2016. [Online]. Available: http://smartvue.com

[21] R. Brunelli, (2009), Template Matching Techniques in Computer Vision: Theory and Practice. Hoboken, NJ, USA: Wiley.

[22] T. Winkler and B. Rinner,( Mar. 2009), "Pervasive smart camera networks exploiting heterogeneous wireless channels,' in Proc. IEEE Int. Conf. Pervasive Comput. Commun. (PerCom), ( pp. 1-4).

[23] D.D.Halland,J.Budinger,( Oct. 2007 ), "Next genats communications, navigation, and surveillance test bed,', in Proc. IEEE/AIAA 26th Digit. Avion. Syst. Conf.,( pp. 4.A.1-1-4.A.1-11).

[24] T. Arulogun, A. Adigun, O. Okediran, and R. Ganiyu, (Jun. 2014 ), "Design and development of a security surveillance system based on wireless sensor network,", Int. J. Innov. Sci., Eng. Technol., vol. 1, no. 4, (pp. 283291).

[25] T. M. Inc. Train a Cascade Object Detector. Accessed: Nov.2014.[Online].Available: http://www.mathworks.se/help/vision/ug/train-acascadeobject-detector.html\#btugex 8

[26] G. Caterina, I. Hunter, and J.Soraghan,( Dec. 2010) “An embedded smart surveillance system for target tracking using a PTZ camera,' in Proc. $4^{\text {th }}$ Eur.Edu.Res. Conf. (EDERC), (pp. 165-169).

[27] A. Hampapur, L. Brown, J. Connell, S. Pankanti, A. Senior, and Y. Tian, (Dec. 2003 ), "Smart surveillance: Applications, technologies and implications,', in Proc. 4th Pacific Rim Conf. Multimedia, Inf., Commun. Signal Process., vol. 2. (pp. 1133-1138).

[28] V. Onut, D. Aldridge, M. Mindel, and S. Perelgut, (2010), "'Smart surveillance system applications,', in Proc. Conf. Center Adv. Stud. Collaborative Res. (CASCON), Riverton, NJ, USA, (pp. 430-432). [Online]. Available: http://dx.doi.org/10.1145/1923947.1924031

[29] AUGUSTO J. V. NETO, ZHONGLIANG ZHAO, JOEL J. P. C. RODRIGUES(Senior Member, IEEE), HUGO BARROS CAMBOIM, AND TORSTEN BRAUN,( February 7, 2018), "Fog-Based Crime-Assistance in Smart IoT Transportation System”.

[30] Karmran Sattar Awaisi,Assad Abbas,Mahdi Zareei,Hasan Ali Khattak et al. (2019),'Towards a Fog Enabled Efficient car parking Architecture",IEEE Access.

[31] Bilal Khalid Dar,Munam Ali Shah, Saif UI Islam, Castren Maple, Shafaq Mussadiq ,Suleman Khan, (2020), “Delay-
Aware Accident Detection and Response System Using Fog Computing", IEEE Acess. 